Note

\title{
Study of Rosemary Peltate Glandular Trichomes Using Combined Morphological
}

\section{and Chemical Approach}

\author{
Takehito Sagawa $^{1,2 *}$, Hidaka IkedA ${ }^{1}$, Tatsuyuki HiraokA ${ }^{1}$ and Kazuichi HayaKawA ${ }^{3}$ \\ ${ }^{I}$ Research and Analysis Center, Research and Product Development Division, S\&B FOODS Incorporated, 38-8 Miyamoto-cho, Itabashi-ku, \\ Tokyo 174-8651, Japan \\ ${ }^{2}$ Graduate School of Natural Science and Technology, Faculty of Pharmaceutical Science, Kanazawa University, Kakuma-machi, \\ Kanazawa, Ishikawa 920-1192, Japan \\ ${ }^{3}$ Institute of Medical, Pharmaceutical and Health Sciences, Kanazawa University, Kakuma-machi, Kanazawa, Ishikawa 920-1192, Japan
}

Received May 8, 2012; Accepted February 16, 2013

Rosemary (Rosmarinus officinalis L.) is a member of the Lamiaceae family that is commonly used in herbal teas. Plants from the Lamiaceae family have numerous peltate glandular trichomes (PGTs). These PGTs contain essential oils, which influence the perception of rosemary aroma. In the present study, we used gas chromatography-mass spectrometry (GC-MS) to evaluate the chemical quantities of 10 active odor compounds from rosemary, i.e., camphene, 1,8-cineole, camphor, linalool, borneol, verbenone, carvone, geraniol, bornyl acetate and alpha-pinene. In addition, we used X-ray computed tomography (X-ray CT) to examine the tissue structure of PGTs from the leaves and stems of rosemary plants. We confirmed that PGTs included 3 types of essential oil. Moreover, we clearly demonstrated that individual PGTs have different structures, filled with different types of essential oil. Our findings suggest that combined use of $\mathrm{X}$-ray CT and GC-MS is an effective means of evaluating the qualities of rosemary PGTs and essential oil.

Keywords: rosemary, X-ray CT, trichome, verbenone, bornyl acetate

\section{Introduction}

Plants belonging to the Lamiaceae family are well-known aromatic potherbs used in herbal teas for their characteristic, favorable aroma. Both fresh and dried potherbs are commonly used. Fresh potherbs are preferred because of the distinct and mild aroma they impart; however, they have a short shelf life. Dried potherbs have a long shelf life, but the perceived aroma frequently differs from that of fresh potherbs as a consequence of the drying process. Therefore, dried potherbs with a perceived aroma similar to that of fresh herbs are desirable.

Plants of the Lamiaceae family are known to have two types of glandular trichome: peltate glandular trichomes (PGTs) and capitate glandular trichomes (CGTs), the presence of which has been demonstrated by morphological and histochemical approaches (Werker, 1993). Scanning electron

*To whom correspondence should be addressed. E-mail: takehito_sagawa@sbfoods.co.jp microscopy has also revealed that PGTs arise as epidermal protuberances (Yamamura et al., 1992; Turner et al., 2000a; Turner et al., 2000b), and staining and chemical analyses of plant tissue sections have shown that PGTs are filled with essential oils, which influence the perception of aromas (Bini et al., 2000; Nik et al., 2004; David ;et al., 2001). PGTs were also the focus of a previous study on essential oil extraction (Iriti et al., 2006). Thus, the essential oil that accumulates in PGTs is thought to be closely related to the perception of potherb aromas, and it is therefore important to consider the chemical and morphological states of the PGTs.

Rosemary (Rosmarinus officinalis L.) is a member of the Lamiaceae family. Rosemary leaves and stems contain many glandular trichomes on the upper and lower surfaces. PGTs and CGTs are distributed on the upper surfaces of leaves, whereas PGTs and glandular hairs (fibrous tissue) are found in close formation on the lower surfaces of the leaves and over the stems.

Preparations such as cryofixation, chemical fixation and 
sectioning are frequently used for histological observation of glandular trichomes (Rodrigues et al., 2008). However, it is difficult to fully elucidate the tissue structure of plant organs using sectioned plant materials. Similarly, it is impossible to microscopically observe the tissue structure of plant materials if the samples are not suitably prepared. Specialized observation methods - such as the use of a micro-slicer image processing system (Do et al., 2004, Ogawa et al., 2001) and small, dedicated magnetic resonance imaging (Koizumi et al., 2006) - are available, but require specially customized instruments.

In the present study, we used a combination of morphological and chemical approaches to evaluate a suitable potherb drying process. Firstly, we used gas chromatographymass spectrometry (GC-MS) to evaluate the chemical qualities of 10 active odor compounds from rosemary. Secondly, we used X-ray computed tomography (X-ray CT) to visualize the appearance and internal tissue structure of PGTs from the leaves and stems of rosemary plants.

\section{Material and Methods}

Plant materials Aerial sections of rosemary plants (Rosmarinus officinalis L.; cultivated in Okinawa, Japan) were purchased from a local wholesale market in Tokyo, Japan. Two types of leaf, the lamina and stipule (Fig. 1), were used for analyses.

Observation of rosemary tissue structure The tissue structure of the rosemary samples was observed by X-ray CT imaging (SMX-100CT-SV3; Shimadzu Kyoto, Japan) and light microscopy (Nikon SMZ745T; Nikon Tokyo, Japan). $\mathrm{X}$-ray CT conditions were as follows: X-ray tube voltage, 40 kv; X-ray tube current, $60 \mu \mathrm{A}$; slice thickness, $0.004 \mathrm{~mm}$; field of view (FOV): XY $2.148 \mathrm{~mm}$ and Z $1.963 \mathrm{~mm}$.

Aroma compounds extracted from leaves and stems

Hot-water extracts were obtained using $2.0 \mathrm{~g}$ of fresh rosemary leaves and stems, with $150 \mathrm{~mL}$ of hot water (temperature $>90^{\circ} \mathrm{C}$ ). Headspace-solid phase micro-extraction (HSSPME) was performed using $2 \mathrm{~mL}$ of each hot-water extract, prior to GC-MS and GC-olfactometry analysis. The HSSPME parameters were as follows: fiber, 50/30 DVB/CAR/

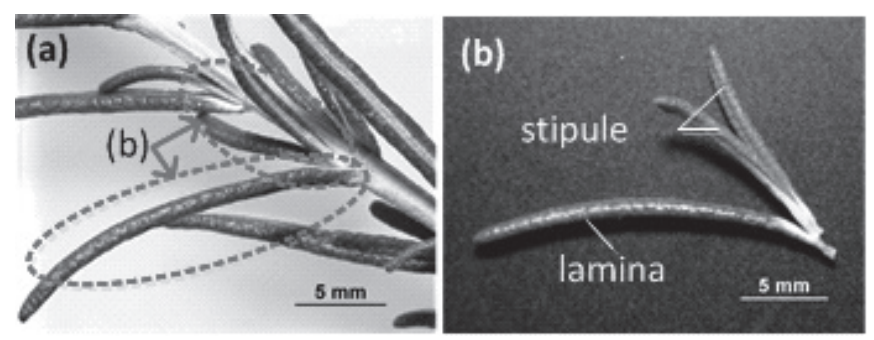

Fig. 1. Images of rosemary (a) leaves and (b) parts of leaves sampled for analyzing peltate glandular trichomes.
PDMS (Spelco Pennsylvania, USA ); absorption conditions, $50^{\circ} \mathrm{C}$ for $10 \mathrm{~min}$ (GC-MS analysis) or $60 \mathrm{~min}$ (GC-olfactometry analysis)

Extraction of aroma compounds from PGTs Essential oils in PGTs were extracted under a microscope using a small piece of filter paper (i.e., small triangular shape, $1 \mathrm{~mm}$ base $\times 5 \mathrm{~mm}$ height). Before extraction, the filter paper was cut with a knife, washed with ethanol, and dried. The extraction procedure used was as follows: (1) a few PGTs were broken using the micro piece of filter paper; (2) PGTs were absorbed onto the filter paper; (3) the filter paper was placed into a 20-mL headspace vial; and (4) HS-SPME followed by GC-MS analysis was performed. HS-SPME parameters were as follows: fiber, 50/30 DVB/CAR/PDMS (Spelco); absorption conditions, $50^{\circ} \mathrm{C}$ for $10 \mathrm{~min}$.

GC-MS analysis GC-MS analysis was performed using a Shimadzu QP-2010 plus system with a Stabilwax fused-silica capillary column $(60 \mathrm{~m} \times 0.25 \mathrm{~mm} \times 0.25 \mu \mathrm{m}$ film thickness; Restec Pennsylvania, USA ). The following conditions were used: oven temperature, $60^{\circ} \mathrm{C}$ for $3 \mathrm{~min}$, increasing at $10^{\circ} \mathrm{C} / \mathrm{min}$ to $100^{\circ} \mathrm{C}$, held at $100^{\circ} \mathrm{C}$ for $1 \mathrm{~min}$, increasing at $3^{\circ} \mathrm{C} / \mathrm{min}$ to $200^{\circ} \mathrm{C}$, held at $200^{\circ} \mathrm{C}$ for $1 \mathrm{~min}$, and increasing at $5^{\circ} \mathrm{C} / \mathrm{min}$ to $230^{\circ} \mathrm{C}$; injector and transfer line temperature, $230^{\circ} \mathrm{C}$; carrier gas, helium with a linear velocity of $30 \mathrm{~cm} /$ $\mathrm{s}$; split ratio, 20:1; SPME desorption time, $1 \mathrm{~min}$; ionization energy, $70 \mathrm{eV}$; scan time, $500 \mathrm{~ms}$; mass range, 40 - 300 aum.

Components were identified based on their retention times and mass spectral fragmentation patterns, which were compared using the Nist05 library and literature reports (Bouteckedjiret et al., 1998; Elamrani et al., 2000; Porte et al., 2000; Serrano et al., 2002). The quantitative values of the individual compounds were estimated from the electronic total ion chromatogram (TIC) peak measurements. Analyses were performed at least in triplicate, and mean values were calculated.

GC-olfactometry analysis GC-olfactometry analysis was performed using a Shimadzu GC-2010 gas chromatograph equipped with a thermal conductive detector (TCD) and a Sniffer 9000 system (Brechbühler Zurich, Switzerland) with a Stabilwax fused-silica capillary column $(60 \mathrm{~m} \times 0.25$ $\mathrm{mm} \times 0.25 \mu \mathrm{m}$ film thickness; Restec). The Sniffer 9000 system was connected directly to the TCD. The following conditions were used: oven temperature, $60^{\circ} \mathrm{C}$ for $3 \mathrm{~min}$, increasing at $10^{\circ} \mathrm{C} / \mathrm{min}$ to $100^{\circ} \mathrm{C}$, held at $100^{\circ} \mathrm{C}$ for $1 \mathrm{~min}$, increasing at $3^{\circ} \mathrm{C} / \mathrm{min}$ to $200^{\circ} \mathrm{C}$, held at $200^{\circ} \mathrm{C}$ for $1 \mathrm{~min}$, and increasing at $5^{\circ} \mathrm{C} / \mathrm{min}$ to $230^{\circ} \mathrm{C}$; carrier gas, helium with a linear velocity of $30 \mathrm{~cm} / \mathrm{s}$; splitless; SPME desorption time, 1 min. Direct intensity method (Delahuntry et al., 2006) of GC-olfactometry analysis was performed by an expert assessor, using a 6-point scale $(0-5)$ of odor intensity (O.I.). 


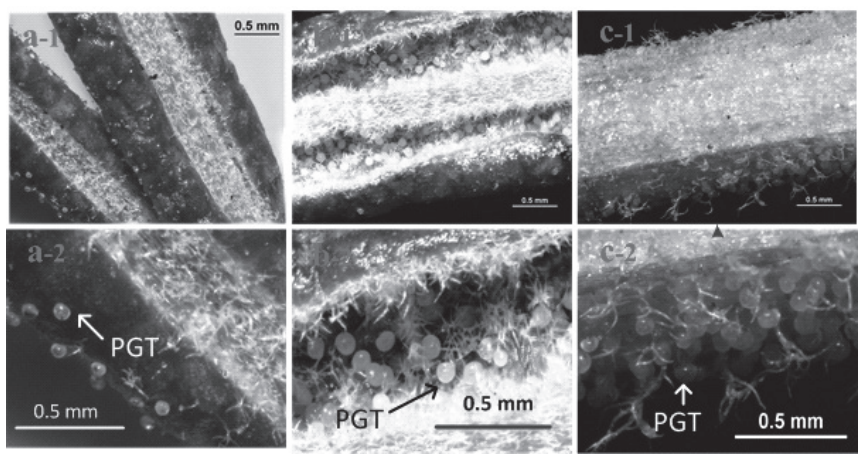

Fig. 2. Images of (a-1) the lower surface of a leaf, (b-1) the upper surface of a leaf, and (c-1) the surface of a stem. (a-2), (b-2), and (c-2) are magnified images of (a-1), (b-1) and (c-1). The white dots over the leaf $(\mathrm{a}-2, \mathrm{~b}-2)$ and stem $(\mathrm{c}-2)$ are peltate glandular trichomes.

When the perception of an odor was strong during GC-olfactometry analysis, an active odor was recognized and the odor intensity was recorded. Each active odor was collected into a headspace vial. Subsequently, qualitative analysis of the collected odors using GC-MS was performed for identification of the active odor compounds, followed by concentration using HS-SPME.

Statistical analysis We performed principal component analysis (PCA), and calculated probable ellipses $(P=0.95)$ by hierarchical cluster analysis with PC1 and PC2 scores, using JMP6 software (SAS Institute)

\section{Results and Discussion}

Light microscopy revealed the presence of PGTs on the leaves and stems of fresh rosemary. PGTs were distributed widely over the stems, and on the upper and lower surfaces of the leaves. GC-MS and GC-olfactometry analysis were performed on hot-water extracts of the leaves and stems, followed by concentration using HS-SPME. Next, it was necessary to detect the presence of active odors and to identify the TIC peaks having odors, because a TIC peak does not necessarily have an odor when using GC-olfactometry analysis. GC-olfactometry analysis was performed 4 times to detect the presence of active odors. Accordingly, we detected the presence of 17 active odors (O.I. 3: 8 odors, O.I. 2: 5 odors, O.I. 1: 4 odors) and identified 10 active odors (O.I. 3: 5 odors, O.I. 2: 3 odors, O.I. 1: 2 odors) as TIC peaks using GC-MS. These 10 peaks were identified as camphene, camphor, linalool, borneol, and carvone (O.I. 3); verbenone, geraniol, and bornyl acetate (O.I. 2); and 1,8-cineole and alpha-pinene (O.I. 1). The sum of the peak areas did not differ significantly between the hot-water extracts of the leaves and stems (data not shown). In contrast, the relative percentages of individual compounds differed significantly between leaf and stem extracts (Fig. 3). Furthermore, the aroma of

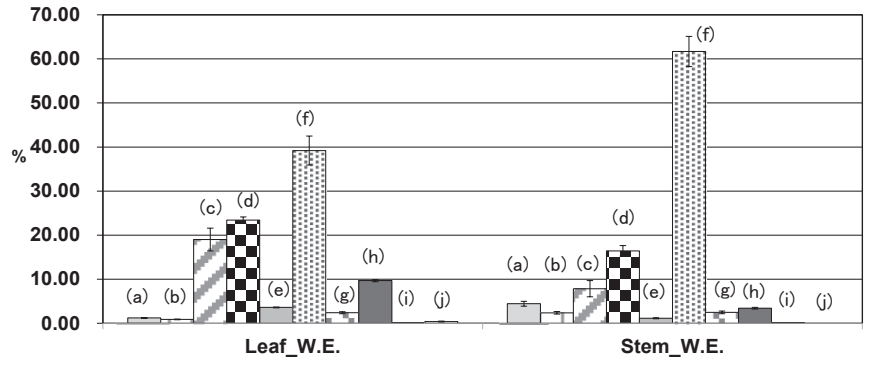

Fig. 3. TIC area ratios of the 10 target compounds derived from the hot water extracts of the leaves and stems. W.E. indicates water extraction. Mean of 3 replicates \pm standard deviation.

(a) $\alpha$-pinene (b) camphene (c) 1,8-cineole (d) camphor (e) linalool (f) bornyl acetate (g) borneol (h) verbenone (i) carvone (j) geraniol. Pecentage of L-carvone: $0.15 \%$ (leaf), $0.12 \%$ (stem). Percentage of geraniol: $0.12 \%$ (leaf), not-identified (stem).
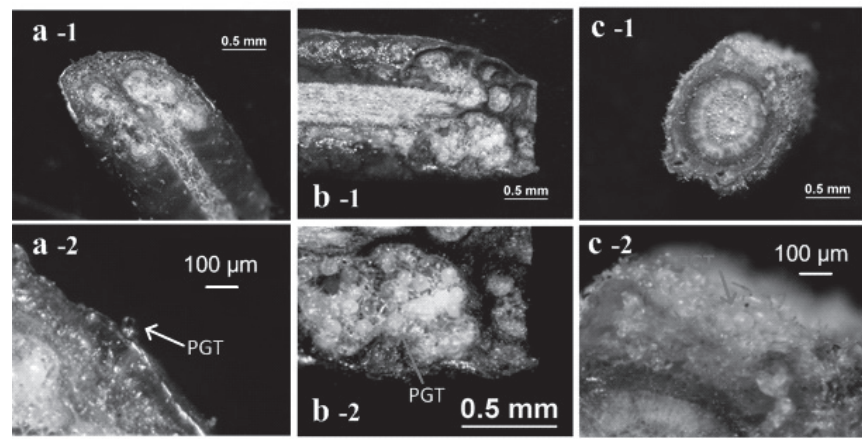

Fig. 4. Images of leaf (a-1, b-1) and stem (c-1) sections. (a-2) is a PGT on the upper surface of a leaf. (b-2) is a PGT on the lower surface of a leaf. (c-2) is a PGT on the surface of a stem.

individual extracts differed between leaves (sweet and cool) and stems (strong cool), suggesting that PGTs in the leaves and stems contain different essential oils.

We investigated the tissue structure of individual PGTs using light microscopy. We observed that PGTs located on the upper surfaces of leaves were distributed over the epidermis (Fig. 4 a-1, a-2). In contrast, PGTs located on the lower surfaces of leaves were distributed on fibrous tissues (Fig. 4 b-1, b-2). PGTs located on the surfaces of stems were found over the epidermis; however, their basal areas rested on fibrous tissues under the epidermis (Fig. 4 c-1, c-2). Next, we used a non-distractive X-ray CT method to reveal the tissue structure of individual PGTs (Fig. 5 a, b). We observed fibrous tissues under the basal areas of PGTs located on the lower surfaces of leaves and on the surface of stems. Meanwhile, PGTs on the upper surfaces of the leaves were distributed over the epidermis (Fig. 6). Our X-ray CT observations complement the light microscopy results. Collectively, our observations suggest the presence of different types of basal tissue under each type of PGT.

$\mathrm{X}$-ray CT is considered to be a useful tool for morphological analysis, because it avoids damage to sample preparations, and provides virtual 3D data. In addition, computer 


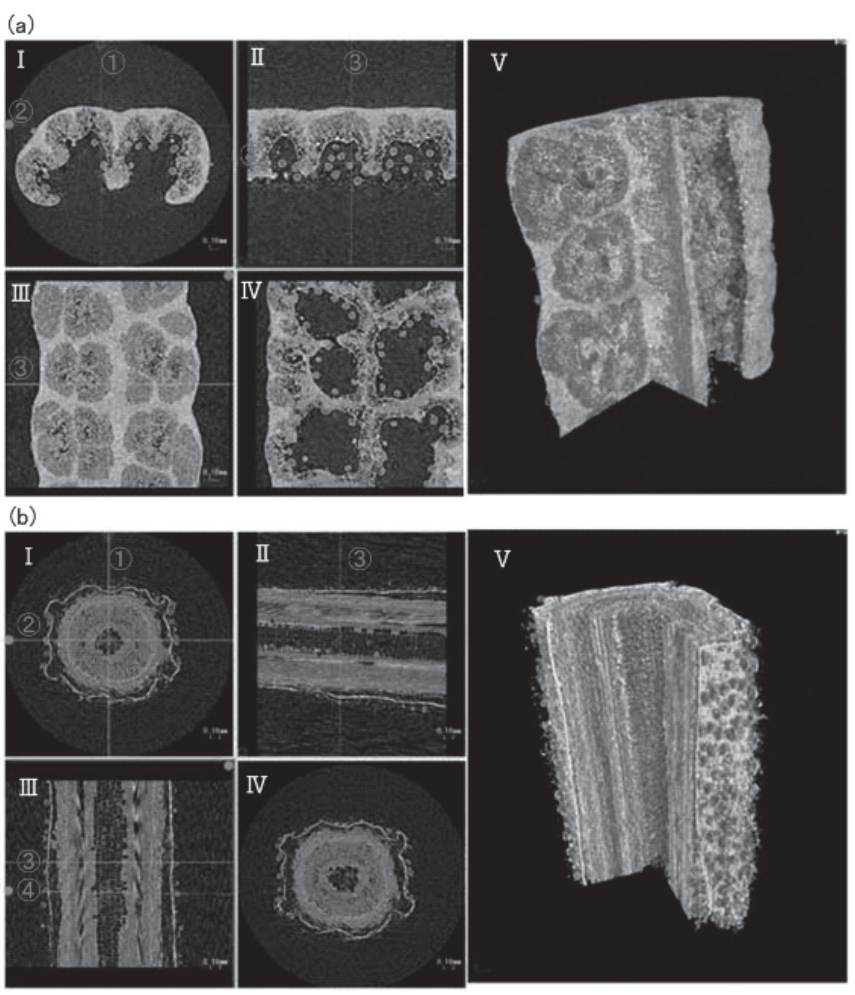

Fig. 5. Multi-planar reconstruction (MPR) and volume rendering (VR) images for rosemary (a) leaf and (b) stem. (I) CT image formed by a cross-section on plane 3; (II) oblique image formed by a cross-section on plane 1; (III) oblique image formed by a crosssection on plane 2; (IV) double-oblique image formed by a crosssection on plane 4; and (V) VR image. Planes 1, 2, and 4 are any arbitrary horizontal or vertical positions used as cross-sections. Plane 3 is the CT position.

storage of CT data simplifies re-analysis of plant morphology.

We subsequently performed GC-MS analysis on each of the essential oils extracted from the PGTs located on the lower and upper surfaces of the leaves, and on the surfaces of the stems. Comparative analysis of the TIC peak area ratios of the compounds identified the active odor compounds (a)

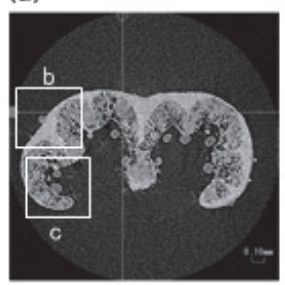

(e)

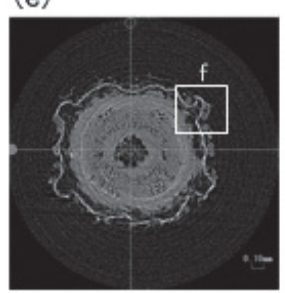

(b)

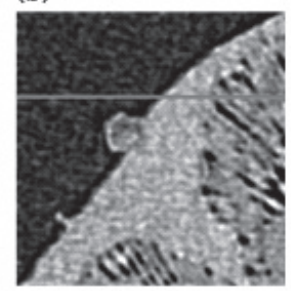

(c)

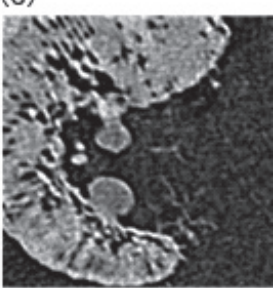

(f)

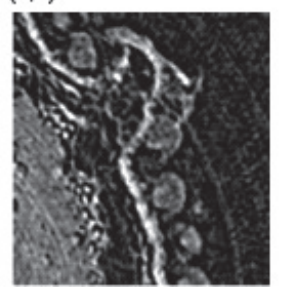

Fig. 6. X-ray CT images of PGTs. (a) A leaf, (b) PGT on the upper surface of a leaf, (c) PGT on the lower surface of a leaf, (e) a stem, and (f) PGT on the surface of a stem.

as alpha-pinene, camphene, 1,8-cineole, camphor, linalool, borneol, bornyl acetate, verbenone, and carvone (Table 1).

Hot-water extracts of the leaves revealed that the PGTs distributed over the surfaces of leaves contained relatively low amounts of geraniol. This finding may be explained by geraniol being present in concentrations below the limit of detection. We performed PCA in relation to the TIC peak area ratios of the essential oils identified in each type of PGT (Fig. 7). We found that, for stipule and lamina, PGTs in the same location (i.e., on the lower or upper surface of a leaf) contained similar essential oils. PGTs located on the lower surfaces of leaves contained different essential oils from those located on the upper surfaces of leaves and on the stems. In addition, PGTs located on the upper surfaces of leaves contained different essential oils from those located on the surface of stems.

Taken together, our GC-MS and PCA data clearly indicated that rosemary PGTs contain 3 types of essential oil - a

Table 1. TIC area ratios of 9 target compounds.

\begin{tabular}{lccrrr}
\hline \multicolumn{1}{c}{ Compound } & U_PGTs leaf(lamina) & U_PGTs leaf (stipule) & L_PGTs leaf (lamina) & L_PGTs leaf (stipule) & PGTs_Stem \\
\hline$\alpha$-pinene & $16.40 \pm 4.17$ & $13.59 \pm 3.88$ & $9.50 \pm 1.95$ & $10.02 \pm 0.38$ & $9.33 \pm 1.85$ \\
camphene & $2.91 \pm 0.33$ & $2.88 \pm 0.21$ & $1.65 \pm 0.25$ & $1.95 \pm 0.30$ & $2.25 \pm 0.35$ \\
1,8-cineole & $9.13 \pm 1.07$ & $9.05 \pm 1.15$ & $12.27 \pm 0.39$ & $15.08 \pm 1.39$ & $5.86 \pm 0.59$ \\
camphor & $8.07 \pm 0.62$ & $10.31 \pm 1.49$ & $20.41 \pm 0.50$ & $18.06 \pm 1.61$ & $17.49 \pm 0.67$ \\
linalool & $1.01 \pm 0.13$ & $1.11 \pm 0.14$ & $4.98 \pm 0.44$ & $4.23 \pm 0.44$ & $2.23 \pm 0.45$ \\
bornyl acetate & $50.37 \pm 2.94$ & $48.21 \pm 4.55$ & $21.96 \pm 2.10$ & $24.29 \pm 1.73$ & $42.81 \pm 1.88$ \\
borneol & $5.95 \pm 1.21$ & $5.97 \pm 1.28$ & $2.34 \pm 0.30$ & $4.07 \pm 0.55$ & $7.56 \pm 1.43$ \\
verbenone & $5.92 \pm 0.81$ & $8.55 \pm 1.73$ & $26.71 \pm 3.25$ & $22.10 \pm 2.15$ & $12.19 \pm 1.88$ \\
carvone & $0.22 \pm 0.01$ & $0.33 \pm 0.12$ & $0.19 \pm 0.04$ & $0.20 \pm 0.01$ & $0.28 \pm 0.04$ \\
\hline
\end{tabular}

Mean of 5 replicates $(\%) \pm$ standard deviation.

U_PGTs leaf: PGTs on the upper surfaces of leaves. L_PGTs leaf: PGTs on the lower surfaces of leaves. 


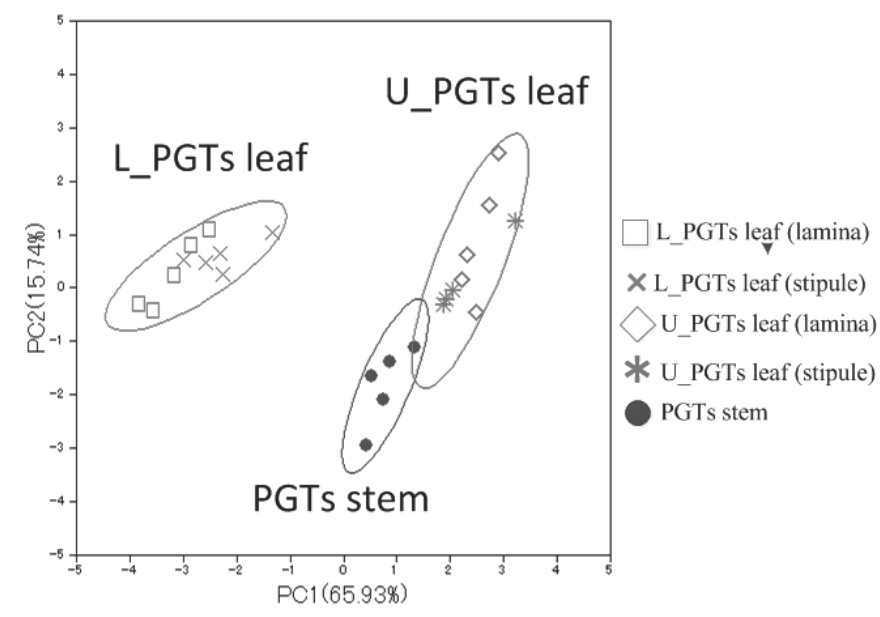

Fig. 7. Principal component analysis score plot of essential oils in each type of PGT. Probable ellipses $(P=0.95)$ were calculated by hierarchical cluster analysis with PC1 and PC2 scores. L_PGTs: peltate glandular trichomes on the lower surfaces of leaves. U PGTs: peltate glandular trichomes on the upper surfaces of leaves. PGT stem: peltate glandular trichomes on the surface of stems.

bornyl acetate type characterized by light cool aroma (PGTs on the upper surfaces of leaves); a verbenone and camphor type characterized by sweet and cool aroma (PGTs on the lower surfaces of leaves); and a bornyl acetate and camphor type characterized by strong cool aroma (PGTs on the surface of stems). We believe that the combined use of GC-MS and X-ray CT is a valuable tool for evaluating the qualities of rosemary aroma.

\section{References}

Bini, L.M., Gentili, L., Trillini, B. and Pellegrino, R. (2000). Secretion constituents of leaf glandular trichomes of Ocimum basilicum L. Flavour Fragrce Chem, pp. 143-150.

Bouteckedjiret, C., Bentrahar, F., Belabbes, R. and Bessiere, J.M. (1998). The essential oil from Rosmarinus officinalis L. in Algeria. J. Essent. Oil Res., 10(6), 680-683.

David, R.G., Jihong, W., Natalia, D., Kyoung, H.N., James, E.S., Efraim, L. and Eran, P. (2001). An investigation of the storage and biosynthesis of phenylpropenes in sweet basil. Plant Physiol., 125, 539-555.

Delahunty, C.M., Eyres, G. and Dufour, J.-P. (2006). Gas chromatography-olfactometry. J. Sep. Sci., 29, 2107-2125.

Do, G.-S., Sagara, Y., Tabata, M., Kudoh, K. and Higuchi, T. (2004). Three-dimensional measurement of ice crystal in frozen beef with a micro-slicer image processing system. Int. J. Refrig., 27, 184190.
Elamrani, A., Zrira, S., Benjilali, B. and Berrada, M. (2000). A study of Moroccan rosemary oils. J. Essent. Oil Res., 12(4), 478495.

Iriti, M., Colnaghi, G., Chemat, F., Smadja, J., Faoro, F. and Visinoni, F.A. (2006). Histo-cytochemistry and scanning electron microscopy of lavender glandular trichomes following conventional and microwave-assisted hydrodistillation of essential oils: a comparative study. Flavour Fregr. J., 21, 704-712.

Koizumi, M., Naito, S., Haishi, T., Utsuzawa, S., Ishida, N. and Kano, H. (2006). Thawing of frozen vegetable observed by a small detected MRI for food research. Magn. Reson. Imaging, 24, 1111-1119.

Nik, Z.B., Mirza, M. and Shahmir, F. (2004). Essential oil of Marrubium cuneatum Russel and its secretory elements. Flavour Fragr. J., 19, 233-235.

Ogawa, Y., Sugiyama, J., Kuensting, H., Ohtani, T., Hagiwara, S., Liu, X., Kokubo, M., Yamamoto, A., Kudoh, K. and Higuchi, T. (2001). Advanced technique for three-dimensional visualization of compound distribution in rice kernel. J. Agric. Food Chem., 49, 736-740.

Porte, A., de O Godoy, R.L., Lopes, D., Kokets, U.M., Goncalves, S.L. and Torquilho, H.S. (2000). Essential oil of Rosemainus officinalis L. (Rosemary) from Rio de Janeiro, Brazil. J. Essent. Oil Res., 12(5), 577-580.

Rodrigues, L., Monteiro, P., Póvoa, O., Teixeira, G., Moldáo, M., Figueiredo, A.C. and Monteiro, A. (2008). Morphology of secretory structures and essential oil composition in Mentha cervina L. from Portugal. Flavour Fregr. J., 23, 340-347.

Serrano, E., Palma, J., Tinoco, T., Venancio, F. and Martins, A. (2002). Evaluation of the essential oils of rosemary (Rosemarinus officinalis L.) from different zones of "Alentejo" (Portugal). J. Essent. Oil Res., 14(2), 87-92.

Turner, G.W., Gershenzon, J. and Croteau, R.B. (2000a). Distribution of peltate glandular trichomes on developing leaves of peppermint. Plant Physiol., 124, 655-663.

Turner, G.W., Gershenzon, J. and Croteau, R.B. (2000b). Development of peltate glandular trichomes of peppermint. Plant Physiol., 124, 665-679.

Werker, E. (1993). Function of essential oil-secreting glandular hair in aromatic plants of the lamiaceae - A Review. Flavour Fragr. J., 8, 249-255

Yamamura, T., Tanaka, S. and Tabata, M. (1992). Localization of the biosynthesis and accumulation of monoterpenoids in glandular trichomes of thyme. Planta Med., 58(2), 153-158. 